\title{
Analytical Solution of the Schrödinger Equation with Spatially Varying Effective Mass for Generalised Hylleraas Potential
}

\author{
Sanjib Meyur, Smarajit Maji, and S. Debnath \\ Department of Mathematics, Jadavpur University, Kolkata 700032, India \\ Correspondence should be addressed to Sanjib Meyur; sanjibmeyur@yahoo.co.in
}

Received 30 May 2014; Revised 19 July 2014; Accepted 21 July 2014; Published 11 August 2014

Academic Editor: Shi-Hai Dong

Copyright ( 2014 Sanjib Meyur et al. This is an open access article distributed under the Creative Commons Attribution License, which permits unrestricted use, distribution, and reproduction in any medium, provided the original work is properly cited. The publication of this article was funded by $\mathrm{SCOAP}^{3}$.

\begin{abstract}
We have obtained exact solution of the effective mass Schrödinger equation for the generalised Hylleraas potential. The exact bound state energy eigenvalues and corresponding eigenfunctions are presented. The bound state eigenfunctions are obtained in terms of the hypergeometric functions. Results are also given for the special case of potential parameter.
\end{abstract}

\section{Introduction}

The study of quantum mechanical systems within the framework of effective position dependent mass has been the subject of much activity in recent years. The Schrödinger equation with position-dependent (nonconstant) mass provides an interesting and useful model for the description of many physical problems. The most extensive use of such an equation is in the physics of semiconductor nanostructures $[1,2]$, quantum dots [3], ${ }^{3} \mathrm{He}$ clusters [4], quantum liquids [5], semiconductor heterostructures $[6,7]$, and so forth.

The solutions of nonrelativistic wave equations with constant mass have been extended to the position dependent mass in recent studies [8-11]. A general formalism for energy spectra and wave functions was found in nonrelativistic problems by using point canonical transformation [8]. Compared to the constant mass wave equation, the position-dependent mass Schrödinger equation is more complex. It is difficult to obtain its analytical solution as usual. Several authors have studied the effects of the position-dependent mass on the solutions of the Schrödinger equation. A positiondependent effective mass, $m(x)=m_{1} \cdot m(x)$, associated with a quantum mechanical particle constitutes a useful model for the study of various potentials such as Morse potential [1218], hard-core potential [18], Scarf potential [19-21], PöschlTeller potential $[22,23]$, spherically ring-shaped potential [24], Hulthén potential [25], Kratzer potential [26], and
Coulomb-like potential $[27,28]$. Different techniques have been developed to obtain its exact solutions, such as factorization methods [29], Nikiforov-Uvarov (NU) methods [30], and supersymmetric quantum mechanics [31]. The positiondependent effective mass might have impact on high-energy physics [31].

The objective of this paper is to investigate the positiondependent effective mass Schrödinger equation for the generalised Hylleraas potential $[32,33]$ by using the NikiforovUvarov (NU) method (Figure 1) [30]. Hylleraas potential is used to describe the interaction between two atoms in a diatomic molecule. We have also investigated the solutions of Hulthén potential and Woods-Saxon potential.

The plan of the present paper is as follows. In Section 2, the Nikiforov-Uvarov method is summarized. Section 3 is devoted to the solution of the position-dependent effective mass Schrödinger equation. In Sections 4 and 5, the Hulthén potential and Woods-Saxon potential are discussed, respectively. The paper is ended with a summary.

\section{Nikiforov-Uvarov Method}

The NU method is a useful technique to solve the secondorder linear differential equations with special orthogonal functions [34]. In this method, after employing an appropriate coordinate transformation $s=s(x)$, the nonrelativistic 


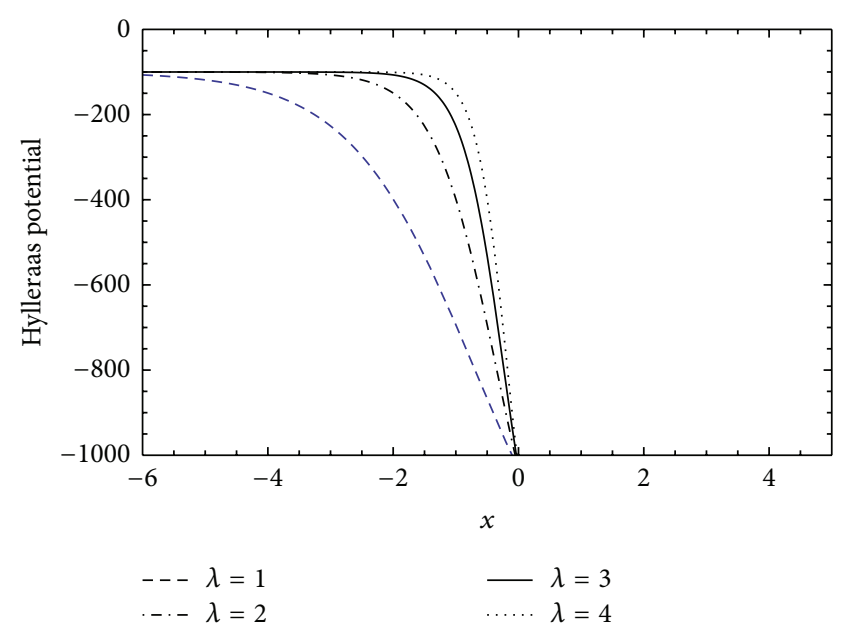

FIGURE 1: Hylleraas potential for $V_{1}=50, V_{2}=100, a=10, b=0.5$, and $d=20$.

Schrödinger equation $d^{2} \psi / d x^{2}+(E-V(x)) \psi=0,(\hbar=2 m=$ 1) can be written in the following form:

$$
\psi^{\prime \prime}(s)+\frac{\tilde{\tau}(s)}{\sigma(s)} \psi^{\prime}(s)+\frac{\tilde{\sigma}(s)}{\sigma^{2}(s)} \psi(s)=0,
$$

where the prime denotes the differentiation with respect to $s$, $\sigma(s)$ and $\tilde{\sigma}(s)$ are polynomials, at most of second degree, and $\tilde{\tau}(s)$ is a polynomial, at most of first degree. In order to obtain a particular solution to (1), we set the following wave function as a multiple of two independent parts:

$$
\psi(s)=\phi(s) y_{n}(s) .
$$

Equation (2) transformed (1) to a hypergeometric-type equation:

$$
\sigma(s) y_{n}^{\prime \prime}(s)+\tau(s) y_{n}^{\prime}(s)+\lambda y_{n}(s)=0,
$$

where first part of $(2), \phi(s)$, has a logarithmic derivative:

$$
\frac{\phi^{\prime}(s)}{\phi(s)}=\frac{\pi(s)}{\sigma(s)},
$$

and second part of (2), $y_{n}(s)$, is the hypergeometric-type function whose polynomial solution satisfies the Rodrigues relation:

$$
y_{n}(s)=\frac{C_{n}}{\rho(s)} \frac{d^{n}}{d s^{n}}\left[\sigma^{n}(s) \rho(s)\right],
$$

where $C_{n}$ is normalization constant and the weight function $\rho(s)$ satisfies the relation as

$$
\frac{d}{d s}[\sigma(s) \rho(s)]=\tau(s) \rho(s) .
$$

The function $\pi(s)$ and the eigenvalue $\lambda$ required in this method are defined as

$$
\begin{gathered}
\pi(s)=\left(\frac{\sigma^{\prime}-\tilde{\tau}}{2}\right) \pm \sqrt{\left(\frac{\sigma^{\prime}-\tilde{\tau}}{2}\right)^{2}-\tilde{\sigma}+k \sigma,} \\
k=\lambda-\pi^{\prime}(s) .
\end{gathered}
$$

Hence, the determination of $k$ is the essential point in the calculation of $\pi(s)$, for which the discriminant of the square root in (7) is set to zero. Also, the eigenvalue equation defined in (8) takes the following new form:

$$
\begin{gathered}
\lambda=\lambda_{n}=-n \tau^{\prime}(s)-\frac{n(n-1)}{2} \sigma^{\prime \prime}(s), \quad n=0,1,2, \ldots, \\
\tau(s)=\widetilde{\tau}(s)+2 \pi(s), \quad \tau^{\prime}(s)<0 .
\end{gathered}
$$

Since $\rho(s)>0$ and $\sigma(s)>0$, the derivative of $\tau(s)$ should be negative [30], which helps to generate the essential condition for any choice of proper bound state solutions. In addition, the energy eigenvalues are obtained from (8) and (9).

\section{Position-Dependent Effective Mass Schrödinger Equation}

In general, working on position-dependent effective mass Hamiltonians is inspired by the von Roos Hamiltonian [35] proposal with $\hbar=2 m_{0}=1$ :

$$
\begin{aligned}
& {\left[-\frac{1}{2}\left(m^{\alpha}(x) \partial_{x} m^{\beta}(x) \partial_{x} m^{\gamma}(x)\right.\right.} \\
& \left.\left.\quad+m^{\gamma}(x) \partial_{x} m^{\beta}(x) \partial_{x} m^{\alpha}(x)\right)+V(x)\right] \varphi(x) \\
& \quad=E \varphi(x)
\end{aligned}
$$

where $\hbar=2 m_{0}=1$ and $m(x)$ is the dimensionless form of the function $m(x)=m_{1} \cdot m(x)$. The ambiguity parameters are constrained by the relation $\alpha+\beta+\gamma=-1$ and we have the following time-independent Schrödinger equation from (11):

$$
H \varphi(x) \equiv\left[-\partial_{x}\left(\frac{1}{m(x)}\right) \partial_{x}+V_{\mathrm{eff}}(x)-E\right] \varphi(x)=0,
$$

where the effective potential is

$$
\begin{aligned}
V_{\mathrm{eff}}(x)= & V(x)+\frac{1}{2}(\beta+1) \frac{m^{\prime \prime}(x)}{m^{2}(x)} \\
& -[\alpha(\alpha+\beta+1)+(\beta+1)] \frac{m^{\prime 2}(x)}{m^{3}(x)},
\end{aligned}
$$

where primes denote derivatives. Thus Schrödinger equation takes the form

$$
\left(-\frac{1}{m(x)} \frac{d^{2}}{d x^{2}}+\frac{m^{\prime}(x)}{m^{2}(x)} \frac{d}{d x}+V_{\mathrm{eff}}(x)-E\right) \varphi(x)=0 .
$$




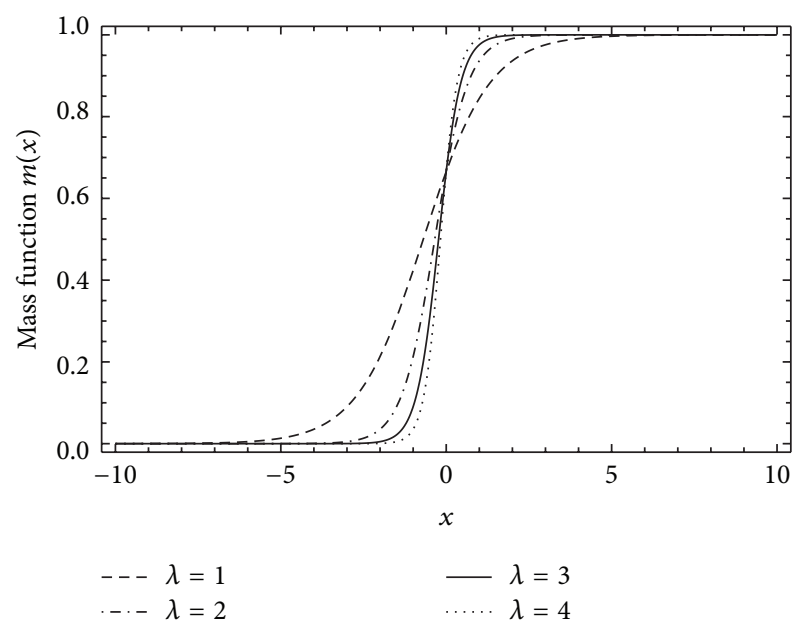

Figure 2: Plot for mass function $m(x)$ for $b=0.5$ and $m_{1}=1$.

Using the transformation [36], $\varphi(x)=m^{\nu}(x) \psi(x)$ in (14), we have

$$
\begin{aligned}
& \left\{-\frac{d^{2}}{d x^{2}}-(2 v-1) \frac{m^{\prime}(x)}{m(x)} \frac{d}{d x}\right. \\
& -(\nu(\nu-2)+\alpha(\alpha+\beta+1)+\beta+1) \frac{m^{\prime 2}}{m^{2}} \\
& \left.+\left(\frac{1}{2}(\beta+1)-v\right) \frac{m^{\prime \prime}(x)}{m(x)}+m(x)(V(x)-E)\right\} \psi(x) \\
& \quad=0,
\end{aligned}
$$

where $V(x)$ is the Hylleraas potential $[29,30]$ given by

$$
V(x)=V_{1} \frac{a+e^{\lambda x}}{b+e^{\lambda x}}-V_{2} \frac{d+e^{\lambda x}}{b+e^{\lambda x}},
$$

where $a(\neq b), b$, and $d(\neq b)$ are the Hylleraas parameters, $V_{1}$, $V_{2}$ are the potential depths, and $-\infty<x<\infty$.

Here, we consider the following mass distribution:

$$
m(x)=\frac{m_{1}}{1+b e^{-\lambda x}}
$$

The most extensive use of such kind of mass is in the physics of semiconductor quantum well structures [11]. The motion of electrons in them can often be described by the envelope function effective-mass Schrödinger equation, where $m_{1}$ is a constant mass (Figure 2).

Obviously it has the exponential form. The above mass function is convergent $m(x) \rightarrow m_{1}$ (finite), when $x \rightarrow \infty$.
In order to reduce the above equation (15) into NikiforovUvarov equation, we make the transformation $s=1 /(1+$ $\left.b e^{-\lambda x}\right),(0 \leq s \leq 1)$ :

$$
\begin{aligned}
A & =\nu(\nu-2)+\alpha(\alpha+\beta+1)+\beta+1, \\
B & =\left(\frac{1}{2}(\beta+1)-\nu\right), \\
\zeta & =\frac{1}{2}-\nu, \\
P-\zeta^{2} & =-A+2 B-\frac{m_{1}(a-b) V_{1}}{b \lambda^{2}}+\frac{m_{1}(d-b) V_{2}}{b \lambda^{2}}, \\
Q-2 \zeta^{2} & =-2 A+3 B-\frac{m_{1}\left(a V_{1}-d V_{2}-b E\right)}{b \lambda^{2}}, \\
R-\zeta^{2} & =-A+B, \\
P-Q+R & =\frac{m_{1}\left(V_{1}-V_{2}-E\right)}{\lambda^{2}}=\varepsilon^{2} ;
\end{aligned}
$$

also

$$
\begin{aligned}
\frac{m^{\prime}(x)}{m(x)} & =\lambda(1-s), \\
\frac{m^{\prime \prime}(x)}{m(x)} & =\lambda^{2}(1-s)(1-2 s) .
\end{aligned}
$$

Using (15)-(19), we have

$$
\begin{aligned}
& \frac{d^{2} \psi}{d s^{2}}+\frac{2 v-(2 v+1) s}{s(1-s)} \frac{d \psi}{d s} \\
& \quad+\frac{1}{s^{2}(1-s)^{2}}\left[\left(\zeta^{2}-P\right) s^{2}+\left(Q-2 \zeta^{2}\right) s+\left(\zeta^{2}-R\right)\right] \psi=0
\end{aligned}
$$

Comparing (20) with (1), we have

$$
\begin{aligned}
& \sigma(s)=s(1-s), \\
& \widetilde{\sigma}(s)=\left(\zeta^{2}-P\right) s^{2}+\left(Q-2 \zeta^{2}\right) s+\left(\zeta^{2}-R\right), \\
& \tilde{\tau}(s)=2 v-(2 v+1) s .
\end{aligned}
$$

Substituting these polynomials into (7), we have

$$
\begin{aligned}
\pi(s)= & \left(\frac{1}{2}-v\right)(1-s) \\
& \pm \begin{cases}(\sqrt{R}-\varepsilon) s-\sqrt{R} ; & k=Q-2 R+2 \varepsilon \sqrt{R} \\
(\sqrt{R}+\varepsilon) s-\sqrt{R} ; & k=Q-2 R-2 \varepsilon \sqrt{R}\end{cases}
\end{aligned}
$$

For physical solutions, it is necessary to choose

$$
\begin{array}{r}
\pi(s)=\zeta(1-s)-(\sqrt{R}+\varepsilon) s+\sqrt{R} \\
\text { if } k=Q-2 R-2 \varepsilon \sqrt{R} .
\end{array}
$$


The origin of the Nikiforov-Uvarov method is negative sign of derivative of $\tau^{\prime}(s)$ because the condition $\tau^{\prime}(s)<0$ helps to generate energy eigenvalues and corresponding eigenfunctions. Therefore $\tau(s)$ becomes

$$
\tau(s)=1+2 \sqrt{R}-2(2+2 \sqrt{R}+2 \varepsilon) s .
$$

Therefore, from (8) and (9), we have

$$
\begin{gathered}
\lambda=Q-2 R-2 \varepsilon \sqrt{R}-\zeta-(\sqrt{R}+\varepsilon), \\
\lambda=\lambda_{n}=2 n(\sqrt{R}+\varepsilon)+n(n+1) .
\end{gathered}
$$

Comparing (25), we have

$$
\begin{gathered}
\sqrt{R}+\varepsilon=-\left(n+\frac{1}{2}\right)+\sqrt{P-v+\frac{1}{4}} \\
\sqrt{R}-\varepsilon=\frac{Q-P}{-(n+1 / 2)+\sqrt{P-v+1 / 4}} .
\end{gathered}
$$

Using (26) and (18), we have

$$
\varepsilon^{2}=\frac{1}{4}\left[2 n+1+2 \sqrt{R}-\sqrt{P-\nu+\frac{1}{4}}\right]^{2} .
$$

Hence the energy becomes

$$
\begin{aligned}
& E_{n} \\
& =-\frac{\lambda^{2}}{4 m_{1}} \\
& \times[2 n+1 \\
& \quad-2 \sqrt{\frac{m_{1}\left(V_{1}-V_{2}\right)}{\lambda^{2}}-\frac{m_{1}\left(V_{1} a-V_{2} d\right)}{b \lambda^{2}}-\alpha(\alpha+\beta+1)} \\
& \left.+2 \sqrt{-\nu(\nu-1)-\alpha(\alpha+\beta+1)-\frac{(\beta+1)}{2}+\left(\frac{1}{2}-\nu\right)^{2}}\right]^{2} \\
& +\left(V_{1}-V_{2}\right), \quad 0 \leq n<\infty .
\end{aligned}
$$

The last term of the square bracket must be positive for BenDaniel and Duke's model [37] $(\alpha=\nu=0, \beta=-1)$ (Figure 3).

From (6), (21), and (24) we obtain the weight function

$$
\rho(s)=s^{2 \sqrt{R}}(1-s)^{2 \varepsilon},
$$

and from (4), (21), and (23) we have

$$
\phi(s)=s^{\sqrt{R}+\zeta}(1-s)^{\varepsilon} .
$$



FIgURE 3: Hylleraas energy $E_{n}$ for $\alpha=0, \beta=-1, a=10, b=0.5$, $d=20, m_{1}=1, V_{1}=50$, and $V_{2}=100$.

Now we use the properties of Jacobi polynomial [35]:

$$
\begin{aligned}
P_{n}^{(\zeta, \xi)}(x)= & \frac{(-1)^{n}(1-x)^{-\zeta}(1+x)^{-\xi}}{2^{n} n !} \\
& \times \frac{d^{n}}{d x^{n}}\left[(1-x)^{n+\zeta}(1+x)^{n+\xi}\right], \\
P_{n}^{(2 \zeta, 2 \xi)}(1-2 s)= & \frac{(-2)^{n}(s)^{-2 \zeta}(1-s)^{-2 \xi}}{2^{n} n !} \\
& \times \frac{d^{n}}{d x^{n}}\left[s^{n+2 \zeta}(1-s)^{n+2 \xi}\right],
\end{aligned}
$$

where $P_{n}^{(a, b)}(x)(a>-1, b>-1)$ is the Jacobi polynomial. The wave functions (Figure 4 ) are obtained from (2), (5), and ((29)-(31)):

$$
\psi_{n}(s)=N_{n} s^{\sqrt{R}+\zeta}(1-s)^{\varepsilon} P_{n}^{(2 \sqrt{R}, 2 \varepsilon)}(1-2 s),
$$

where $N_{n}$ is normalization constant to be determined from the normalization condition:

$$
\int_{-\infty}^{\infty}\left|\psi_{n}(x)\right|^{2} d x=1=\int_{0}^{1}\left|\psi_{n}(s)\right|^{2} d s
$$

For acceptable solution it is required that $|\sqrt{R}+\zeta| \geq \varepsilon$ when $\sqrt{R}+\zeta<0, \varepsilon>0$ and $\sqrt{R}+\zeta \leq|\varepsilon|$ when $\sqrt{R}+\zeta>0, \varepsilon<0$.

\section{Hulthén Potential}

We set the conditions $V_{2}=V_{1}, a=1+d$, and $b=-q$; the potential in (16) reduces to Hulthén potential (Figure 5) [38-41]:

$$
V(x)=-V_{1} \frac{e^{-\lambda x}}{1-q e^{-\lambda x}} .
$$




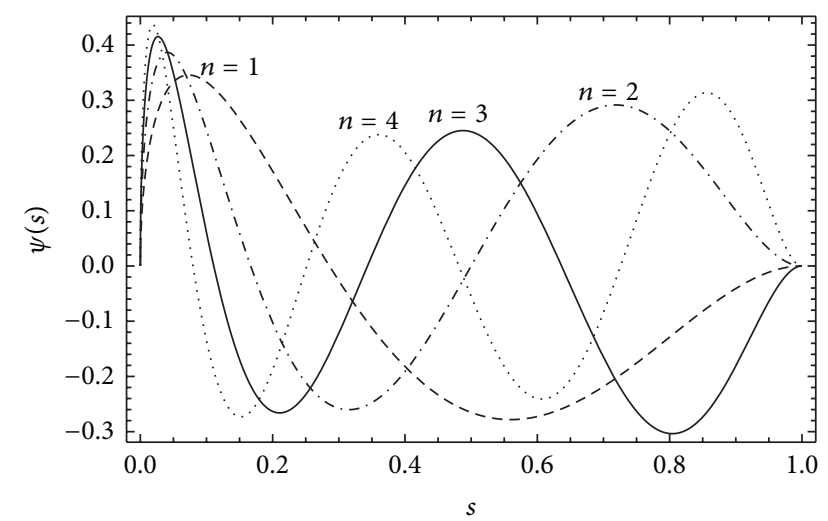

FIGURE 4: Wave functions $\psi_{n}(s)$ for $\alpha=0, \beta=-1, v=1 / 2$, and $\varepsilon=2$.

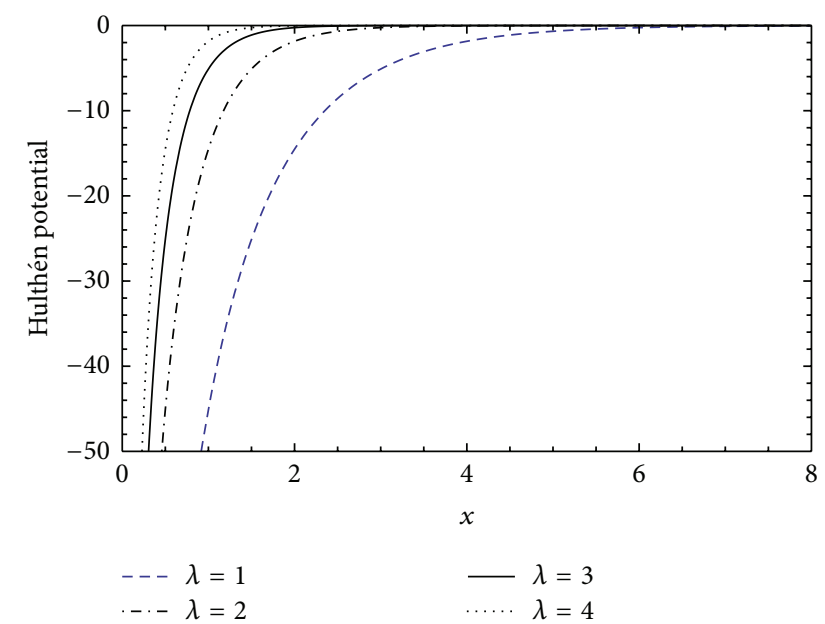

FIGURE 5: Hulthén potential for $V_{1}=100.0$ and $q=0.5$.

Then the energy becomes

$$
\begin{aligned}
& E_{n} \\
& =-\frac{\lambda^{2}}{4 m_{1}} \\
& \quad \times\left[2 n+1-2 \sqrt{\frac{m_{1} V_{1}}{q \lambda^{2}}-\alpha(\alpha+\beta+1)}\right. \\
& \left.\quad+2 \sqrt{-\nu(\nu-1)-\alpha(\alpha+\beta+1)-\frac{(\beta+1)}{2}+\left(\frac{1}{2}-\nu\right)^{2}}\right]^{2}
\end{aligned}
$$

with $0 \leq n<\infty$. It is exactly the same result in the literature [38] for $m_{1}=1$.

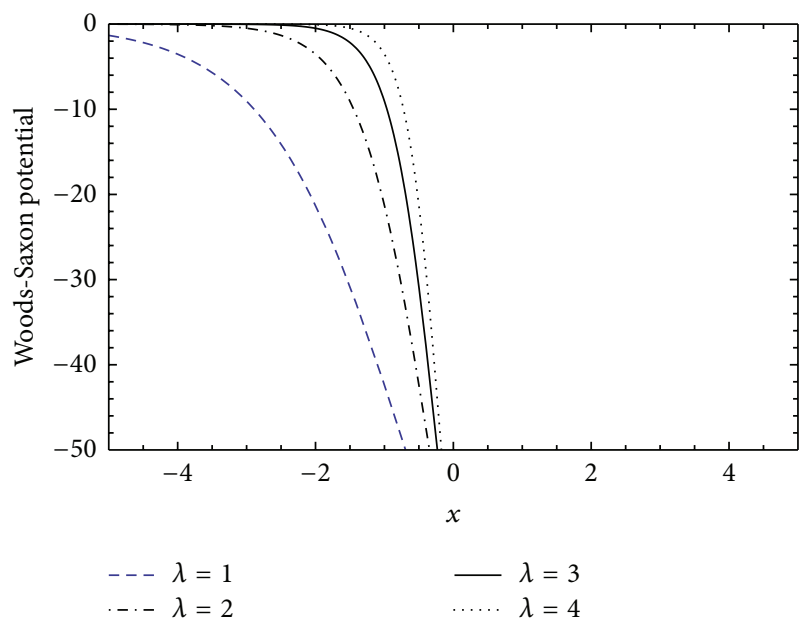

Figure 6: Woods-Saxon potential for $V_{0}=100.0$ and $b=0.5$.

\section{Woods-Saxon Potential}

For the conditions $V_{1}=-V_{0}, a=0$, and $V_{2}=0$, the potential given in (16) becomes Woods-Saxon potential (Figure 6) [4045],

$$
V(x)=-V_{0} \frac{1}{1+b e^{-\lambda x}} .
$$

Then the energy becomes

$E_{n}$

$=-\frac{\lambda^{2}}{4 m_{1}}$

$\times\left[2 n+1-2 \sqrt{\frac{m_{1}(1-b) V_{0}}{b \lambda^{2}}-\alpha(\alpha+\beta+1)}\right.$

$\left.+2 \sqrt{-\nu(\nu-1)-\alpha(\alpha+\beta+1)-\frac{(\beta+1)}{2}+\left(\frac{1}{2}-\nu\right)^{2}}\right]^{2}$

$-V_{0}$,

where $0 \leq n<\infty$.

\section{Conclusion}

We have applied the NU method derived for the exponentialtype potentials to obtain the bound state solutions of the effective Schrödinger equation with position-dependent mass for the Hylleraas potential. Furthermore, a suitable choice of a position mass function of the exponential-like form has also been devised. Also we have shown that our results are consistent with ones obtained before. 


\section{Conflict of Interests}

The authors declare that there is no conflict of interests regarding the publication of this paper.

\section{Acknowledgment}

The authors wish to deliver their sincere gratitude to the referee for constructive suggestions and technical comments on the paper.

\section{References}

[1] G. Bastard, Wave Mechanics Applied to Semiconductor HeteroStructures, EDP Sciences, Les Editions de Physique, Les Ulis, France, 1992.

[2] P. Harrison, Quantum Wells, Wires and Dots, John Wiley \& Sons, New York, NY, USA, 2000.

[3] L. Serra and E. Lipparini, "Spin response of unpolarized quantum dots," Europhysics Letters, vol. 40, no. 6, pp. 667-672, 1997.

[4] M. Barranco, M. Pi, S. M. Gatica, E. S. Hernández, and J. Navarro, "Structure and energetics of mixed ${ }^{4} \mathrm{He}-{ }^{3} \mathrm{He}$ drops," Physical Review B, vol. 56, no. 14, pp. 8997-9003, 1997.

[5] F. Arias De Saavedra, J. Boronat, A. Polls, and A. Fabrocini, "Effective mass of one $\mathrm{He}^{4}$ atom in liquid $\mathrm{He}^{3}$," Physical Review $B$, vol. 50, no. 6, pp. 4248-4251, 1994.

[6] I. Galbraith and G. Duggan, "Envelope-function matching conditions for GaAs/(Al,Ga)As heterojunctions," Physical Review B, vol. 38, no. 14, pp. 10057-10059, 1988.

[7] C. Weisbuch and B. Vinter, Quantum Semiconductor Heterostructure, Academic Press, New York, NY, USA, 1993.

[8] L. Dekar, L. Chetouani, and T. F. Hammann, "Wave function for smooth potential and mass step," Physical Review A, vol. 59, no. 1, pp. 107-112, 1999.

[9] A. D. Alhaidari, "Solution of the Dirac equation with positiondependent mass in the Coulomb field," Physics Letters A, vol. 322, no. 1-2, pp. 72-77, 2004.

[10] B. Roy and P. Roy, "A Lie algebraic approach to effective mass Schrödinger equations," Journal of Physics. A. Mathematical and General, vol. 35, no. 17, pp. 3961-3969, 2002.

[11] B. Gönul, D. Tutcu, and O. Özer, "Supersymmetric approach to exactly solvable systems with position-dependent effective masses," Modern Physics Letters A, vol. 17, no. 31, pp. 2057-2066, 2002.

[12] S. M. Ikhdair, "Rotation and vibration of diatomic molecule in the spatially-dependent mass Schrödinger equation with generalized q-deformed Morse potential," Chemical Physics, vol. 361, no. 1-2, pp. 9-17, 2009.

[13] C. Tezcan, R. Sever, and O. Yesiltas, "A new approach to the exact solutions of the effective mass Schrodinger equation," International Journal of Theoretical Physics, vol. 47, no. 6, pp. 1713-1721, 2008.

[14] A. Arda and R. Sever, "Bound state solutions of the Schrödinger for generalized Morse potential with position-dependent mass," Communications in Theoretical Physics, vol. 56, no. 1, pp. 51-54, 2011.

[15] G. Lévai and O. Özer, "An exactly solvable Schrödinger equation with finite positive position-dependent effective mass," Journal of Mathematical Physics, vol. 51, no. 9, Article ID 092103, 2010.
[16] B. Bagchi, C. Quesne, and R. Roychoudhury, "PseudoHermiticity and some consequences of a generalized quantum condition," Journal of Physics A, vol. 38, no. 40, pp. L647-L652, 2005.

[17] J. Yu, S.-H. Dong, and G.-H. Sun, "Series solutions of the Schrödinger equation with position-dependent mass for the Morse potential," Physics Letters A, vol. 322, no. 5-6, pp. 290297, 2004.

[18] S.-H. Dong and M. Lozada-Cassou, "Exact solutions of the Schrödinger equation with the position-dependent mass for a hard-core potential," Physics Letters A: General, Atomic and Solid State Physics, vol. 337, no. 4-6, pp. 313-320, 2005.

[19] O. Mustafa and S. H. Mazharimousavi, "First-order intertwining operators with position dependent mass and $\eta$-weakpseudo-Hermiticity generators," International Journal of Theoretical Physics, vol. 47, no. 2, pp. 446-454, 2008.

[20] H. Motavali, "Bound state solutions of the Dirac equation for the Scarf-type potential using Nikiforov-Uvarov method," Modern Physics Letters A, vol. 24, no. 15, pp. 1227-1236, 2009.

[21] A. P. Zhang, P. Shi, Y. W. Ling, and Z. W. Hua, "Solutions of one-dimensional effective mass Schrödinger equation for PTsymmetric Scarf potential," Acta Physica Polonica A, vol. 120, no. 6, pp. 987-991, 2011.

[22] M. G. Miranda, G. Sun, and S.-H. Dong, "The solution of the second Pöschl-Teller-like potential by Nikiforov-Uvarov method," International Journal of Modern Physics E, vol. 129, no. 1, pp. 123-129, 2010.

[23] H. Hassanabadi, E. Maghsoodi, S. Zarrinkamar, and H. Rahimov, "Dirac equation for generalized Pöschl-Teller scalar and vector potentials and a Coulomb tensor interaction by Nikiforov-Uvarov method," Journal of Mathematical Physics, vol. 53, no. 2, Article ID 022104, 2012.

[24] M. C. Zhang, G. H. Sun, and S.-H. Dong, "Exactly complete solutions of the Schrödinger equation with a spherically harmonic oscillatory ring-shaped potential," Physics Letters A, vol. 374, no. 5, pp. 704-708, 2010.

[25] F. Yasuk and M. K. Bahar, "Approximate solutions of the Dirac equation with position-dependent mass for the Hulthén potential by the asymptotic iteration method," Physica Scripta, vol. 85, no. 4, Article ID 045004, 2012.

[26] S. M. Ikhdair and R. Sever, "Exactly solvable effective mass $D$-dimensional Schrödinger equation for pseudoharmonic and modified Kratzer problems," International Journal of Modern Physics C, vol. 20, no. 3, pp. 361-372, 2009.

[27] R. Koç and M. Koca, "A systematic study on the exact solution of the position dependent mass Schrödinger equation," Journal of Physics A: Mathematical and General, vol. 36, no. 29, pp. 81058112, 2003.

[28] S. Ortakaya, "Pseudospin symmetry in position-dependent mass dirac-coulomb problem by using laplace transform and convolution integral," Few-Body Systems, vol. 54, no. 11, pp. 2073-2080, 2013.

[29] S.-H. Dong, Factorization Method in Quantum Mechanics, Springer, New York, NY, USA, 2007.

[30] A. F. Nikiforov and V. B. Uvarov, Special Functions of Mathematical Physics, Birkhäauser, Basel, Switzerland, 1988.

[31] B. Midya, B. Roy, and T. Tanaka, "Effect of position-dependent mass on dynamical breaking of type $\mathrm{B}$ and type $X_{2} \mathcal{N}$-fold supersymmetry," Journal of Physics A: Mathematical and Theoretical, vol. 45, no. 20, Article ID 205303, 22 pages, 2012. 
[32] E. A. Hylleraas, "Energy formula and potential distribution of diatomic molecules," Journal of Chemical Physics, vol. 3, article 595, no. 9, 1935.

[33] A. N. Ikot, "Solution of Dirac equation with generalized hylleraas potential," Communications in Theoretical Physics, vol. 59, no. 3, pp. 268-272, 2013.

[34] M. Abramowitz and I. Stegun, Handbook of Mathematical Function with Formulas, Graphs and Mathematical Tables, Dover, New York, NY, USA, 1964.

[35] O. von Roos, "Position-dependent effective masses in semiconductor theory," Physical Review B, vol. 27, no. 12, pp. 7547-7552, 1983.

[36] R. Sever, C. Tezcan, Ö. Yeşiltaş, and M. Bucurgat, "Exact solution of effective mass Schrödinger equation for the Hulthen potential," International Journal of Theoretical Physics, vol. 47, no. 9, pp. 2243-2248, 2008.

[37] D. J. BenDaniel and C. B. Duke, "Space-charge effects on electron tunneling," Physical Review, vol. 152, no. 2, pp. 683-692, 1966.

[38] L. Hulthén, "Über die eigenlösungen der schrödinger-gleichung der deuterons," Arkiv för Matematik A, vol. 28, no. 5, pp. 1-12, 1942.

[39] S. Meyur and S. Debnath, "Real spectrum of non-Hermitian Hamiltonians for hulthén potential," Modern Physics Letters A, vol. 23, no. 25, pp. 2077-2084, 2008.

[40] O. Aydoğdu, A. Arda, and R. Sever, "Scattering of a spinless particle by an asymmetric Hulthén potential within the effective mass formalism," Journal of Mathematical Physics, vol. 53, no. 10, Article ID 102111, 2012.

[41] D. S. Saxon and R. D. Woods, "Diffuse surface optical model for nucleon-nuclei scattering," Physical Review, vol. 95, no. 2, pp. 577-578, 1954.

[42] A. Arda and R. Sever, "Bound states of the Klein-Gordon equation for Woods-Saxon potential with position dependent mass," International Journal of Modern Physics C, vol. 19, no. 5, pp. 763-773, 2008.

[43] C. Berkdemir, A. Berkdemir, and R. Sever, "Polynomial Solution of the Schrödinger equation for the generalized Woods-Saxon potential," Physical Review C, vol. 74, no. 3, Article ID 039902, 2006.

[44] O. Aydoğdu, A. Arda, and R. Sever, "Effective-mass Dirac equation for Woods-Saxon potential: scattering, bound states, and resonances," Journal of Mathematical Physics, vol. 53, Article ID 042106, 2012.

[45] O. Aydoğdu and R. Sever, "Pseudospin and spin symmetry in the Dirac equation with Woods-Saxon potential and tensor potential," European Physical Journal A, vol. 43, no. 1, pp. 73-81, 2009. 

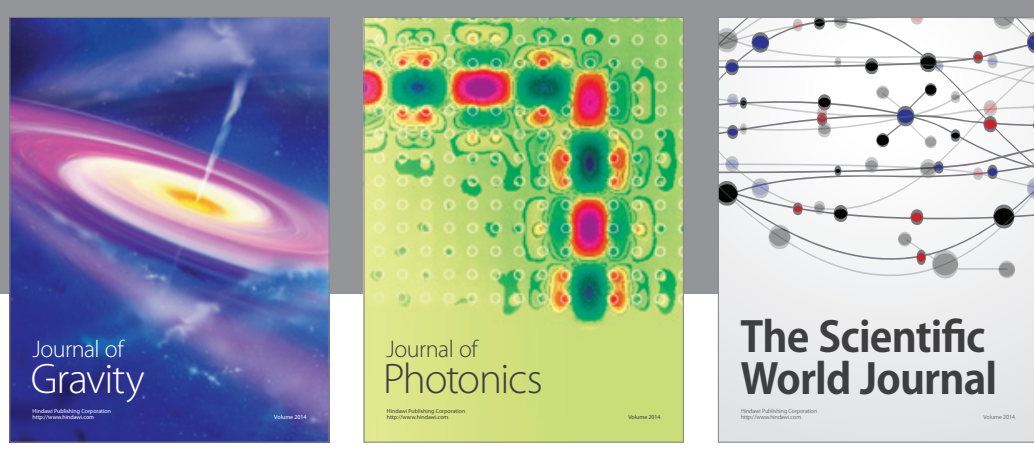

The Scientific World Journal
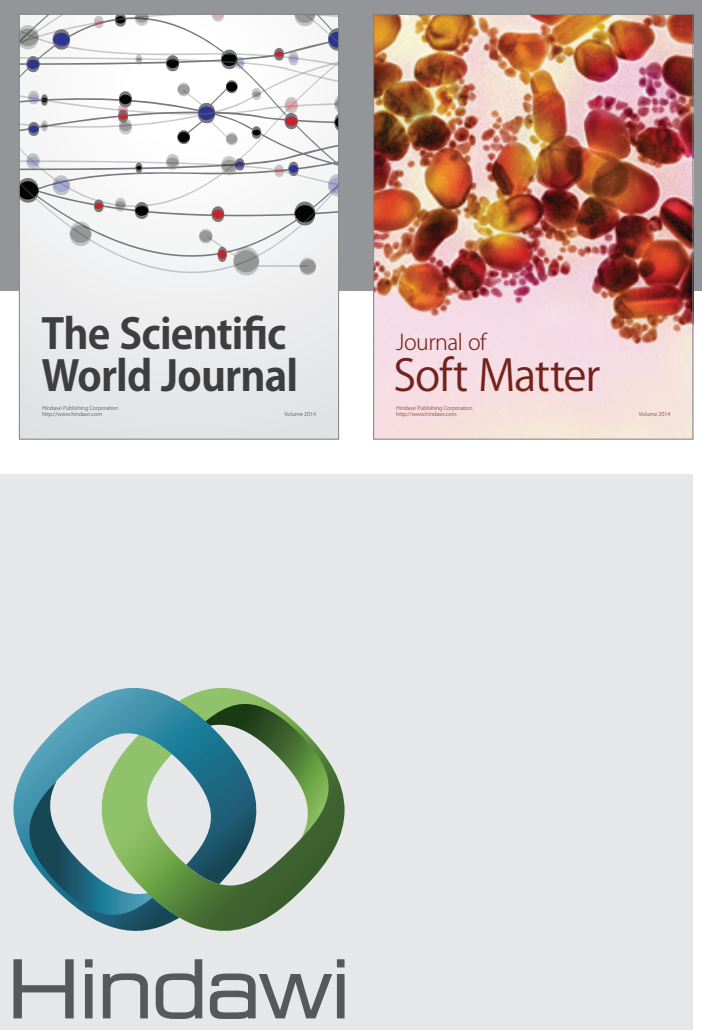

Submit your manuscripts at

http://www.hindawi.com

nternational Journal of

Statistical Mechanics
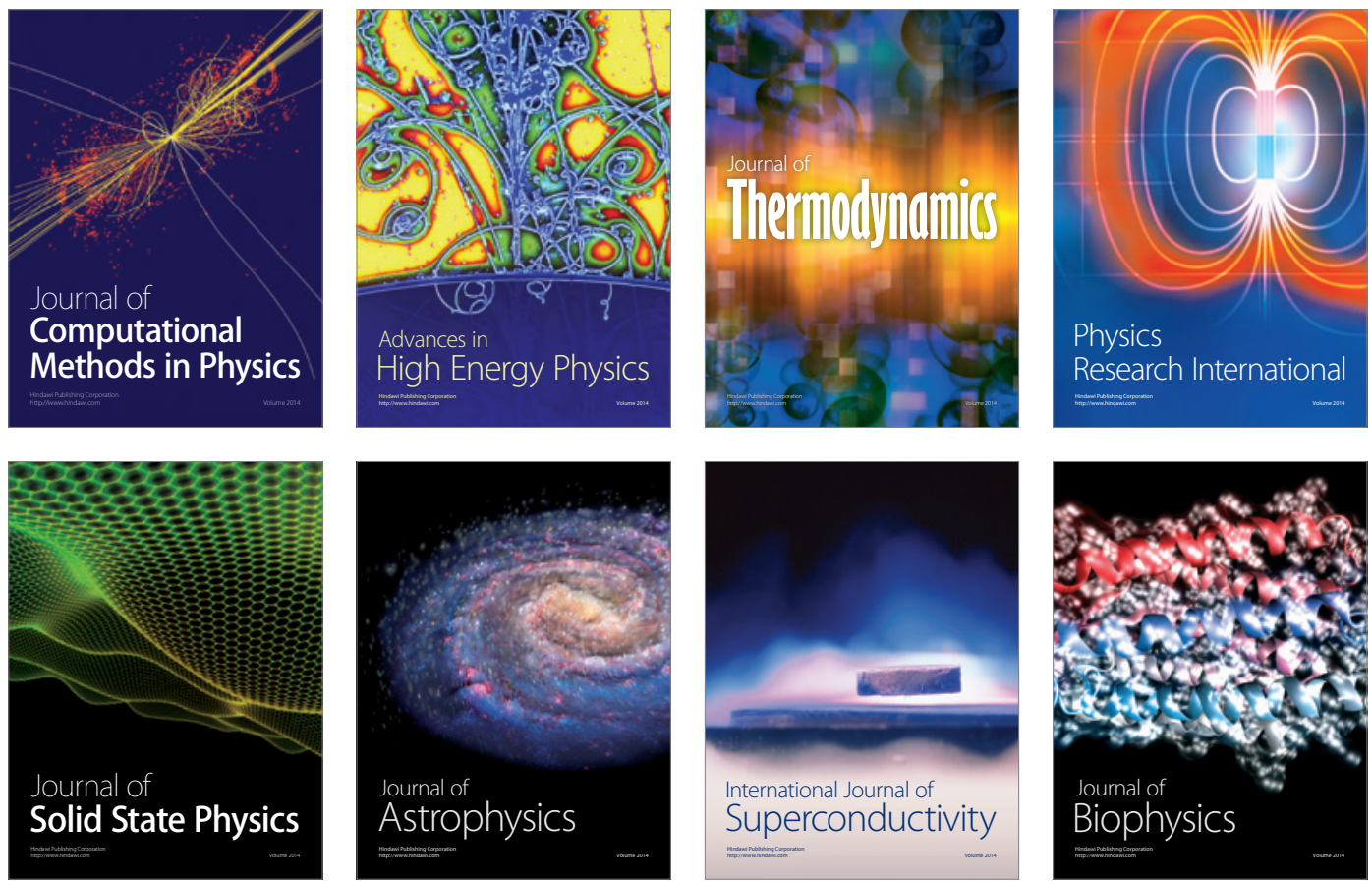
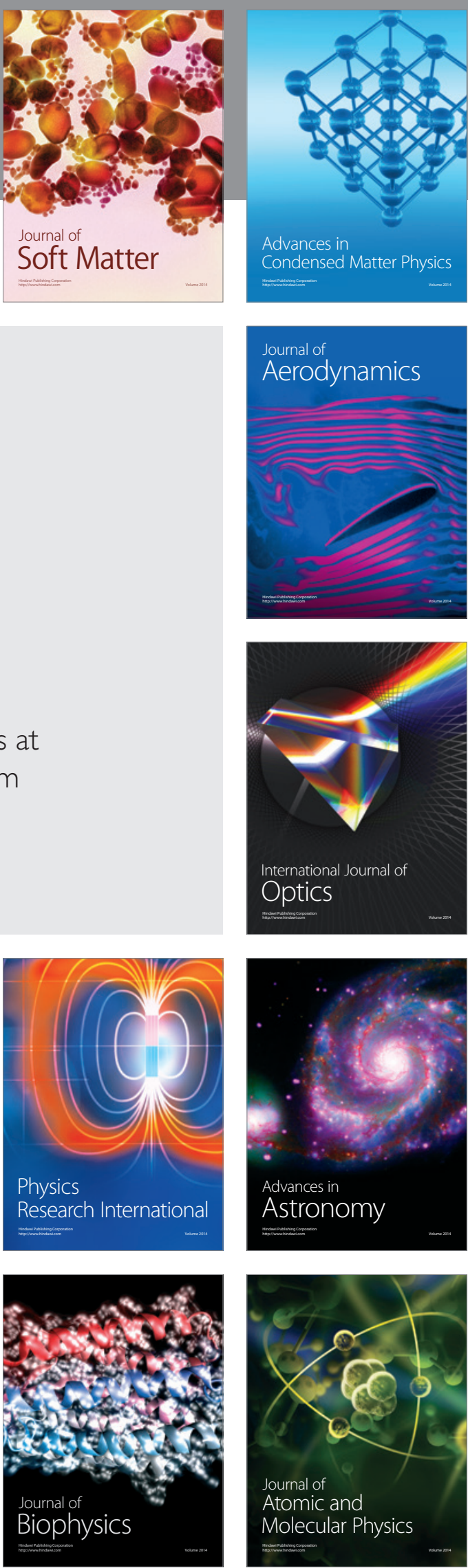